\title{
Estudio del Perfil de Aminoácidos y Análisis Proximal de Pastas Secas Extruidas a Base de Harina de Quinua y Harina de Chontaduro
}

\author{
Saúl Dussán-Sarria ${ }^{(1)^{*}}$, Raquel E. De la Cruz-Noguera(1) y Sandra P. Godoy(2) \\ (1) Facultad de Ingeniería y Administración, Departamento de Ingeniería, Universidad Nacional de Colombia. Sede \\ Palmira. A.A. 237. Palmira, Valle del Cauca-Colombia. (e-mail: sdussan@unal.edu.co; reden@unal.edu.co) \\ (2) Facultad de Ciencias Agrarias, Departamento de Agroindustria, Universidad del Cauca, Popayán, Cauca-Colombia. \\ (e-mail: sgodoy@unicauca.edu.co)
}

* Autor a quien debe ser dirigida la correspondencia

Recibido Feb. 6, 2019; Aceptado Mar. 27, 2019; Versión final Jun. 11, 2019, Publicado Dic. 2019

\section{Resumen}

El objetivo de este trabajo, fue identificar a partir de una pasta extruida a base de harinas no convencionales como la harina de quinua y chontaduro, sus composiciones proximales y sus perfiles de aminoácidos. El estudio del análisis proximal y perfil de aminoácidos esenciales se realizó de acuerdo a las metodologías referenciadas en la literatura. Durante el proceso termomecánico de extrusión de las harinas evaluadas se observó una pérdida de las propiedades nutricionales de las pastas secas y hubo disminución en los contenidos de minerales, grasa total y aminoácidos esenciales. Las pastas con harina de quinua presentaron una pérdida significativa de aminoácidos y en particular de lisina, seguramente causada por el proceso de extrusión y debida a la reacción de Maillard. La mezcla de sémola de trigo, harinas de quinua y chontaduro es la pasta seca que se perfila como la alternativa más adecuada frente a las pastas comerciales.

Palabras clave: Chenopodium quinoa Will; Bactris gasipaes Kunthes; harina de quinoa; harina de chontaduro

\section{Study of the Amino Acid Profile and Proximal Analysis of Extruded Dry Pastas based on Quinoa Flour and Peach Palm Flour}

\begin{abstract}
The aim this job was to identify an extruded pasta based on unconventional flours such as quinoa flour and peach palm flour, their proximal compositions and their amino acid profiles. The study of the proximal analysis and profile of essential amino acids was carried out according to methodologies described in the literature. During the thermomechanical process of extrusion of flours, losses of nutritional properties of dried pasta were observed and also a decrease in the content of minerals, total fat and essential amino acids. The quinoa flour pasta had a significant loss of amino acids, particularly of lysine, probably caused by the extrusion process and the Maillard reaction. The mixture of wheat semolina, quinoa flours and peach palm flour is the dry pasta that is emerging as the most adequate alternative to commercial pastas.
\end{abstract}

Keywords: Chenopodium quinoa Willd; Bactris gasipaes Kunthes; quinoa flour; peach palm flour 


\section{INTRODUCCIÓN}

Una de las grandes problemáticas a nivel mundialy nacional, especialmente en la zona pacifica colombiana,es la falta de alimentos con características nutricionales apropiadas que contribuyan a mejorar la calidad de vida,especialmente en la población infantil y anciana, las cuales presentan altas tasas de desnutrición(DNP, 2018). Esta situación impulsa investigaciones que permiten el desarrollo de nuevos productos con mejores contenidos nutricionales en los alimentos.Colombia ha avanzadopoco en el desarrollo de investigaciones que contemplan el uso de harinas, que permitan la sustitución parcial de subproductos a base de trigo en la elaboración de productos de panificación, galletería, pastelería y pastas, debido a las deficiencias en la calidad nutricional de este cereal (Astaizaet al., 2010). Diferentes investigadores han reportado trabajos desarrolloscon pastas elaboradas a partir de mezclas de sémola de trigo con otras harinas como cebada, frijol, yuca, amaranto, garbanzo, habas, maíz, arroz, y quinua, entre otros, evaluando parámetros de calidad como pruebas de cocción, pruebas microbiológicas y aceptación de los consumidores.La quinua (Chenopodium quinoa Willd) es un cereal y el chontaduro (Bactris gasipaes Kunthes) el fruto de una palmera, ambos productos agrícolas producidos en Colombia, con excelentes atributos sensoriales y nutricionales entre los cuales se destacan carbohidratos, ácidos grasos, vitaminas, minerales y aminoácidos esenciales como la luteína y la lisina (Murphy y Matanguihan, 2015).Estos atributos de la quinua y el chontaduro, los torna potencialmente adecuados para substituir el trigo en algunos derivados como las pastas alimenticias. La fortificación de alimentos es empleada para prevenir la deficiencia de algunos nutrientes o añadir un efecto saludable a los alimentos (Serpa-Guerra et al., 2017).

En la agroindustria de alimentos, y en este caso, la agroindustria de las pastas alimenticias, la principal operación de proceso es la extrusión, que consiste en un proceso termomecánico con transferencia de calor, transferencia de masa, cambios de presión y corte, originando efectos como la cocción, esterilización, secado, texturización, hinchado, mezclado, amasado y formato (Singh, 2007). La extrusión ofrece la posibilidad de modificar las propiedades funcionales de los ingredientes alimentarios y / o texturizarlos, al igual que puede tener efectos beneficiosos e indeseables en el valor nutricional del producto final. Los efectos beneficiosos incluyen la gelatinización del almidón, la destrucción de factores anti nutricionales, el aumento de las fibras dietéticas solubles, la reducción de la oxidación de los lípidos y la contaminación de microorganismos, permite conservar los colores y sabores naturales de los alimentos. Además, el proceso de extrusión desnaturaliza las enzimas indeseables y esteriliza el producto terminado (Singh, 2007; Nikmaram et al., 2015).Las desventajas del proceso de extrusión son la aparición de la reacción de Maillard que lleva a reducir el valor nutricional de la proteína y la pérdida de vitaminas lábiles al calor. La extrusión requiere un control cuidadoso si se desea mantener la calidad del producto. Mantener y aumentar la calidad nutricional de los alimentos durante el procesamiento de alimentos siempre esun área potencialmente importante para la investigación (Singh, 2007; Nikmaram et al., 2015; Serpa et al., 2017).

Este trabajo tuvo como objetivo obtener una pasta seca extruida con mejores propiedades nutricionales a partir de diferentes niveles de inclusión de harina de quínoa y chontaduro, creando de esta forma una alternativa a las pastas secas comerciales y a su vez un aporte a la revisión de literatura en el área de propiedades nutricionales de pastas alimenticias fortificadas.

\section{MATERIALES Y MÉTODOS}

La metodologíase presenta en 5 subsecciones, local y materia prima, preparación de las mezclas, extrusión de las pastas, análisis proximal, perfil de aminoácidos, análisis microbiológico y análisis estadístico de los datos.

\section{Local y materia prima}

El trabajo se llevó a cabo en el Laboratorio de Frutas y Hortalizas de la Universidad Nacional de Colombia, Sede Palmira (1000 msnm) y el Laboratorio de Vegetales de la Universidad del Cauca (1738 msnm). El chontaduro y la quinua fueron obtenidos en el Departamento del Cauca, Colombia. Fue utilizada la variedad Blanca Jericó para obtener la harina de quinua, para ello fue realizado el secado del grano hasta un contenido de humedad entre el $10 \%$ y $12 \%$ bs. El proceso de desaponificación húmeda se efectuó mediante el lavado del grano con agua que a través de movimientos giratorios dentro de un recipiente, generando fricción entre ellos, liberando así la primera capa del grano permitiendo el desprendimiento de las saponinas, posteriormente el grano pasa a ser secado.. La molienda se llevó a cabo en un molino industrial hasta obtener harina con tamaño de partícula de 0,5 mm (Tamiz No. 35 - US). La variedad Rojo Cauca fue utilizada para obtener de la harina de chontaduro, El fruto entero de chontaduro se sometió al escaldado con agua en ebullición durante 45 minutos. Una vez cocido se retiró cáscara y semilla, y la pulpa fue sometida al secado en un horno convectivo (T: $61 \pm 2{ }^{\circ} \mathrm{C}, 4,5 \mathrm{~h}, \overline{\mathrm{u}}: 3 \pm 0,5 \mathrm{~m} / \mathrm{s}$, HR: $41 \pm 3 \%$ ), posterior al 
secado, se realizó la molienda en seco en molino industrial para obtener tamaño de partícula de 0,5 mm (Tamiz No. 35 - US).

En la sémola de trigo fueron considerados los lineamientos de calidad exigidos para el producto según la Norma Técnica NTC 420 (ICONTEC, 2015a), se utilizó sémola de trigo durumobtenida de trigo duro adquirida en la empresa Segalco S.A del Departamento del Cauca. En el huevo fueron considerados los lineamientos de calidad exigidos para el producto según la Norma Técnica NTC 6116 (ICONTEC, 2015b),se utilizó huevo deshidratado y pasteurizado proporcionado por la empresa Ovopacific S.A.S. La adición de huevo en la elaboración de las pastas tiene como objetivo conferir calidad, mejorar características de color amarillo brillante y mejorar propiedades mecánicas de la masa durante la extrusión.

\section{Preparación de las mezclas}

Las pastas fueron obtenidas a partir de diferentes mezclas de las harinas sémola de trigo (ST), harina de quinua $(\mathrm{HQ})$ y harina de chontaduro $(\mathrm{HCh})$. El mezclado y homogenización de las harinas se realizó en una mezcladora semi-industrial marca KITCHEN AID a velocidad media (120rpm) por 10 minutos, adicionando en esta etapa lentamente el agua destilada con la ayuda de un aspersor, obteniendo así una mezcla homogénea. La pasta control correspondió a la elaboración de las pastas comerciales con el total de harina de sémola de trigo y se denominó ST, las demás pastas fueron obtenidas sustituyendola sémola de trigo por diferentes contenidos de harina de quinua y harina de chontaduro. En la formulación el $100 \%$ de la pasta se consideró como fracción seca, es decir, a la suma de las cantidades de las harinas, sin embargo, para poder realizar la extrusión de las 4 formulaciones, la fracción liquida fue constante y consistió en $30 \mathrm{~g} / 100 \mathrm{~g}$ de agua destilada y $5 \mathrm{~g} / 100 \mathrm{~g}$ de huevo. La descripción y denominación de cada pasta o tratamiento se observa en la Tabla 1.

Tabla1: Denominación y descripción de las formulaciones de pastas secas ( $\mathrm{g} / 100 \mathrm{~g}$ de muestra seca). ST: sémola de trigo (control); STHQ: sémola de trigo y harina de quinua; STHCh: sémola de trigo y harina de chontaduro; STHQHCh: sémola de trigo, harina de quinua y harina de chontaduro.

\begin{tabular}{|c|c|c|c|c|}
\hline Ingrediente & ST & STHQ & STHCh & STHQHch \\
\hline ST & 100 & 70 & 70 & 50 \\
\hline HQ & 0 & 30 & 0 & 30 \\
\hline HCh & 0 & 0 & 30 & 20 \\
\hline
\end{tabular}

\section{Extrusión de las pastas}

Todas las mezclas fueron sometidas a un proceso de extrusión para obtener las pastas. Fue utilizado el extrusor de doble tornillo sin fin modelo DS 32 -II (Jinan Saixin Machinery co, China) programado aun perfil de temperatura de $50^{\circ} \mathrm{C}$, el motor principal a una velocidad de giro de $480 \mathrm{rpm}$ y una velocidad de alimentación de $540 \mathrm{rpm}$, fue utilizada una boquilla de salida o dado de $5 \mathrm{~mm}$, las condiciones de extrusión fueron constantes para el procesamiento de todas las mezclas (Manthey et al., 2004).Las pastas extruidas fueron sometidas a un proceso de secado colocándolas en reposo en bandejas de acero inoxidable y utilizando un secador marca BINDER de doble cabina con convección de aire, programado a $45^{\circ} \mathrm{C}$ durante 12 horas, enseguida se dejaron enfriar durante 1 hora y se empacaron en bolsas de polietileno de alta densidad con cierre hermético y así quedaron dispuestas las pastas secas para los respectivos análisis.

\section{Análisis proximal}

El análisis proximal se realizó en las dos harinas y en las cuatro pastas evaluadas. Los resultados se obtuvieron por triplicado. El contenido de humedad se determinó siguiendo el procedimiento descrito en la norma NTC 529 (ICONTEC, 2009), contenido de proteínas según la norma ISO 1871 (ISO, 2009), contenido de cenizas con la norma AOAC 923.03 (AOAC, 2005), contenido de grasa total según la norma AOAC 922.06 (AOAC 2012), contenido de fibra cruda según la norma NTC 668 (ICONTEC, 1973) y contenido de carbohidratos por diferencia al $100 \%$.

\section{Perfil de aminoácidos}

El perfil de aminoácidos fue realizado a las dos harinas y en la cuatro pastas se casa través de High performance liquid chromatography (HPLC) en un equipo de marca Agilent, modelo 1100, detector UV/VIS, volumen de inyección de $5 \mu \mathrm{l}$, flujo $2 \mathrm{ml} / \mathrm{min}$, fase móvil con solución bufferr de monofosfato de sodio y $\mathrm{pH}$ 7.8 , y solución de acetonitrilo $45 \%$, metanol $45 \%$ y agua $45 \%$, a una porción de muestra se le realizo el proceso de hidrolisis establecido de acuerdo a la norma AOAC 994.12 (AOAC, 1997). La solución final se inyecto siguiendo la metodología descrita en la nota de análisis "Amino Acid Analysis Using Zorbax Eclipse AAA columns and the Agilent 1110 HPLC".Los análisis se realizaron por triplicado. 


\section{Análisis microbiológico}

Este análisis fue realizado por laboratorio externo para cuantificar los recuentos de mohos, levaduras, estafilococos, escherichia coli, bacillus cereus, salmonella y esporas de clostridium de acuerdo a las normas estandarizadas para cada caso. Los análisis se realizaron por triplicado.

\section{Análisis estadístico}

Se realizó un análisis completamente al azar y ANOVA unifactorial para conocer la diferencia entre los valores de los resultados presentados en el análisis proximal y perfil de aminoácidos de los cuatro tratamientos y la comparación de medias entre ellos a través de la prueba de rango múltiple Duncan con un valor de significancia del $95 \%(p<0,05)$. Se evaluó con el programa estadístico Minitab v. 16. Igualmente se reportó el valor de la desviación estándar de las repeticiones $( \pm S D)$.

\section{RESULTADOS Y DISCUSIÓN}

A continuación se presentan los resultados y discusión de las características de las pastas extruidas como lo son el análisis proximal, el perfil de aminoácidos, el análisis microbiológico y además se discute la posible influencia del proceso de extrusión sobre estas características.

\section{Análisis proximal}

En la Tabla 2,se observan los resultados del análisis proximal de las harinas, en donde la harina de quinua variedad Blanca Jericó tiene mayor contenido de proteína, cenizas y fibra, comparada con la harina de chontaduro variedad Rojo Cauca e igual contenido de carbohidratos $(p<0,05)$. En contraste, la harina de chontaduro tiene mayor contenido de grasa total, resultados similares encontraron De Oliveira et al.(2006)reportando valores en el análisis químico de la harina de pejibaye procedente de California y Brasil humedad de $7,08 \%$, cenizas $1,49 \%$, proteína $4,23 \%$, lípidos $5,88 \%$, carbohidratos $83,81 \%$.

Tabla 2. Valores de contenidos proximales de las harinas ( $\mathrm{g} / 100 \mathrm{~g}$ de muestra). ST: harina de sémola de trigo duro; HQ: harina de quinua; HCh: harina de chontaduro. $\pm S D$.

\begin{tabular}{|c|c|c|c|c|c|c|}
\hline Harina & Humedad & Proteína & Cenizas & Grasa Total & $\begin{array}{c}\text { Fibra } \\
\text { cruda }\end{array}$ & Carbohidratos \\
\hline ST & $11,01 \pm 0,23^{\mathrm{a}}$ & $13,30 \pm 0,32^{\mathrm{a}}$ & $1,1 \pm 0,10^{\mathrm{a}}$ & $1,70 \pm 0,20^{\mathrm{a}}$ & $2,40 \pm 0,33^{\mathrm{a}}$ & $77,60 \pm 2,0^{\mathrm{a}}$ \\
\hline $\mathrm{HQ}$ & $9,00 \pm 0,21^{\mathrm{b}}$ & $14,58 \pm 0,80^{\mathrm{b}}$ & $2,6 \pm 0,21^{\mathrm{b}}$ & $4,01 \pm 0,41^{\mathrm{b}}$ & $1,39 \pm 0,21^{\mathrm{b}}$ & $69,81 \pm 2,20^{\mathrm{b}}$ \\
\hline $\mathrm{HCh}$ & $11,54 \pm 0,27^{\mathrm{c}}$ & $4,50 \pm 0,51^{\mathrm{c}}$ & $1,8 \pm 0,22^{\mathrm{c}}$ & $8,73 \pm 0,32^{\mathrm{c}}$ & $0,10 \pm 0,01^{\mathrm{c}}$ & $73,43 \pm 1,51^{\mathrm{b}}$ \\
\hline
\end{tabular}

La harina de la sémola de trigo durum presenta un contenido de proteínas similar a la harina de quinua. Las proteínas presentes en harinas y pastas evidencian la presencia de aminoácidos (Maldonado, 2010). En cuanto al contenido de humedad, las 3 harinas presentan un valor inferior al $15,5 \%$, es decir, son adecuadas para la elaboración de pastas según la norma NTC 420 (ICONTEC, 2015a).En la Tabla 3 se observan los resultados del análisis proximal de las pastas secas evaluadas. Todos los valores medios del contenido de humedad, contenido de proteína, contenido de ceniza y grasa total de las pastas evaluadas son diferentes $(p<0,05)$. La pasta formadapor sémola de trigo, harinas de quinua y chontaduro (STHQHCh) presentó el menor valor de contenido de humedad, con un valor de 7,58 $\pm 0,06 \%$ y la pasta de sémola de trigo (ST o control) el mayor valor, con un valor de $9,06 \pm 0,05 \%$. Por lo tanto, todas las pastas secas evaluadas presentaron un contenido de humedad menor a $13 \%$, lo que las hace adecuadas para su almacenamiento según la norma NTC 1055 (ICONTEC, 2007).

Tabla 3. Valores del análisis proximal de las pastas extruidas ( $\mathrm{g} / 100 \mathrm{~g}$ de muestra). ST: sémola de trigo (control); STHQ: sémola de trigo y harina de quinua; STHCh: sémola de trigo y harina de chontaduro; STHQHCh: sémola de trigo, harina de quinua y harina de chontaduro. Diferentes letras en la misma columna indican diferencias estadísticas según prueba de Duncan $(p<0,05)$. ND: No detectada. $\pm S D$.

\begin{tabular}{|c|c|c|c|c|c|c|}
\hline Pasta & Humedad & Proteína & Cenizas & Grasa Total & Fibra cruda & Carbohidratos \\
\hline ST & $9,06 \pm 0,05^{\mathrm{a}}$ & $13,86 \pm 0,06^{\mathrm{a}}$ & $0,65 \pm 0,02^{\mathrm{a}}$ & $3,63 \pm 0,02^{\mathrm{a}}$ & ND & $72,80 \pm 0,02^{\mathrm{a}}$ \\
\hline STHQ & $8,19 \pm 0,04^{\mathrm{b}}$ & $15,68 \pm 0,04^{\mathrm{b}}$ & $1,15 \pm 0,02^{\mathrm{b}}$ & $4,89 \pm 0,03^{\mathrm{b}}$ & ND & $70,09^{\mathrm{a}} \pm 0,08^{\mathrm{b}}$ \\
\hline STHCh & $7,85 \pm 0,03^{\mathrm{c}}$ & $12,19 \pm 0,05^{\mathrm{c}}$ & $0,92 \pm 0,02^{\mathrm{c}}$ & $6,24 \pm 0,05^{\mathrm{c}}$ & ND & $72,80 \pm 0,03^{\mathrm{a}}$ \\
\hline STHQHCh & $7,58 \pm 0,06^{\mathrm{d}}$ & $14,72 \pm 0,05^{\mathrm{d}}$ & $1,20 \pm 0,01^{\mathrm{d}}$ & $6,12 \pm 0,01^{\mathrm{d}}$ & ND & $70,38^{0} \pm 0,07^{\mathrm{b}}$ \\
\hline
\end{tabular}

La pastas secas de STHQ y STHQHCh presentaron los mayores contenidos de proteína y por consiguientede aminoácidos, con contenidos de proteína de 15,68 $\pm 0,04 \%$ y $14,72 \pm 0,05 \%$ 
respectivamente. Estos valoresson debidos al aporte proteínico proporcionado por la harina de quinua, mientras que en las pastas ST y STHCh es debido al aporte proteínico de la sémola de trigo (Tabla 1). La pasta elaborada con sémola de trigo y harina de chontaduro fue la que presentó el menor valor de proteína con un valor de 12,19 \pm 0,05\%. Maldonado (2010) yDe Oliveiraet al. (2006) comentan que los contenidos de proteína en la harina de quinua son superiores a las harinas de cereales como trigo y maíz. De acuerdo a la norma colombiana NTC 1055 (ICONTEC, 2007), para pastas secas el porcentaje mínimo de contenido de proteínas debe ser de $10,5 \%$, es decir, las pastas evaluadas cumplen con este requerimiento nutricional.

La pasta que presentó el mayor contenido de cenizas fue la pasta STHQHCh con un valor de 1,20 $\pm 0,01 \%$ y la de menor contenido fue la pasta ST con un valor de 0,65 $\pm 0,02 \%$. Al igual que el contenido de proteínas, las pastas con presencia de quinua exhibieron los mayores aportes en minerales representados en contenido de cenizas. Las pastas extruidas a base de quinua, es decir, STHQ y STHQHCh, las cuales tenían la misma proporción de harina de quinua en la formulación, exhibieron contenidos de minerales de $1,15 \pm 0,02 \mathrm{~g} / 100 \mathrm{~g}$ de muestra y 1,20 $\pm 0,01 \mathrm{~g} / 100 \mathrm{~g}$ de muestra respectivamente, valores inferiores a los encontradosinicialmente en la harina de quinua que fue de 2,6 $\pm 0,21 \mathrm{~g} / 100 \mathrm{~g}$ de muestra. Seguramente la disminución del contenido nutricional debido a los minerales se debe al efecto del proceso de extrusión. Cerón-Fernandez et al. (2016) encontraron también una disminución significativa del contenido de cenizas en pastas extruidas a base de quinua variedad Tunkahuan. Considerando la norma NTC 1055 (ICONTEC, 2007) que indica que las pastas secas alimenticias deben presentar un valor máximo de cenizas de $1,2 \%$, todas las pastas evaluadas cumplirían con este requerimiento.

Las pastas elaboradas con harina de chontaduro presentan los mayores contenidos de grasa total, la pasta STHCh arrojandoun valor de 6,24g/100g demuestra, seguida de la pasta STHQHCh con 6,12g/100g de muestra, mientras que el menor contenido de grasa total lo mostro la pasta sémola de trigo (ST) con 3,63 \pm $0,02 \mathrm{~g} / 100 \mathrm{~g}$ de muestra $(\mathrm{p}<0,05)$. El fruto de chontaduro presentó aproximadamente $5,88 \mathrm{~g} / 100 \mathrm{~g}$ de muestra de grasas (De Oliveiraet al., 2006) con un alto porcentaje de grasas insaturadas en su mayor contenido ácido oleico (Restrepo et al., 2012).La pasta seca STHCh tuvo mayor proporción de harina de chontaduro en la formulación y probablementeesto incidió en un mayor valor de grasa, sin embargo, se evidencia una disminución en el contenido de grasa considerando el valor inicial en la harina de chontaduro que fue de $8,73 \pm 0,32 \mathrm{~g} / 100 \mathrm{~g}$ de muestra. En pastas extruidas a base de quinua variedad Tunkahuan, Cerón-Fernández et al. (2016) también encontraron una disminución del contenido de grasa total. Liting et al. (2018) también encontraron una reducción de ácidos grasos insaturados de aceite de palma debido al proceso de extrusión y consideran que la temperatura en la extrusión y el contenido de humedad en el proceso provocan la reducción de estos ácidos grasos. Las pastas secas con inclusión de harina de quinua (STHQ Y STHQHCh) presentaron valores similares en el contenido de carbohidratos, 70,09 \pm 0,08 g/100 g de muestra y 70,38 \pm $0,07 \mathrm{~g}$ de muestra $(\mathrm{p}<0,05)$. Lo mismo sucede entre las otras dos pastas con un contenido un poco mayor. Cerón-Fernándezet al. (2016) encontraron un valor similar, 69,10 g/100 g de muestra, en pastas extruidas con dos variedades diferentes de quinua. En general, las 4 pastas secas evaluadas presentaron un importante aporte calórico representado por sus elevados contenidos de carbohidratos (De Oliveiraet al.,2006).

La no presencia de fibra en las pastas, se debe posiblemente, al efecto de la extrusión, provocado por la elevada presión y temperatura ocasionando el rompimiento de los carbohidratos de mayor tamaño presentes en las harinas, tornándolos de menores tamaños, los cuales son más solubles en agua (CerónFernándezet al., 2016). El proceso de cocción por extrusión incluye el tratamiento mecánico y térmico del material a alta presión y alta temperatura provocadas por el movimiento del tornillo sin fin del extrusor. La energía producida en forma de calor durante el proceso de cocción por extrusión se debe al calor energético del tornillo como efecto de cizallamiento, la energía utilizada para aumentar la presión de la masa viscosa, la energía cinética generada en el espacio entre un tornillo y un barril, también, la energía creada durante los cambios fisicoquímicos de los materiales en el tiempo de proceso en extrusor(Wójtowiez, 2012). Las fuerzas de corte creadas por la acción giratoria de los tornillos, junto con las fuerzas de fricción, compresión y presión proporcionan el ambiente necesario para una cocción rápida y transformando los ingredientes en una pasta viscoelástica (Firibu, 2011).

\section{Perfil de Aminoácidos}

En la Tabla 4 y Tabla 5 se observan los valores de los contenidos de 7 de los 10 aminoácidos consideradosesenciales en la nutrición humana (Chito-Trujillo et al. 2017)en cada una de las harinas y pasta evaluadas respectivamente. La harina de quinua presenta contenidos de aminoácidos considerablemente mayores que la harina de chontaduro $(p<0,05)$ (Tabla 4). Por esta razón a la quinua se le considera un supercereal, por su elevado contenido de proteínas y sualto valor nutricional atribuido al contenido de aminoácidos esenciales (Mao et al., 2014; Murphy y Matanguihan, 2015).En general, la pasta STHQ (sémola de trigo más harina de quinua) presentó el mayor contenido de todos los aminoácidos (Tabla 5), 
guardando relación con la pasta con mayor contenido de proteína (Tabla 4). La pasta seca STHQHCh (sémola de trigo + harina de quinua + harina de chontaduro), en general, presentó altos contenidos de aminoácidos y a su vez una elevada cantidad de proteína (Tabla 3 y Tabla 4).

Tabla 4. Contenido de aminoácidos esenciales en las harinas de quinua y chontaduro ( $\mathrm{g} / 100 \mathrm{~g}$ de muestra). Diferentes letras minúsculas en la misma columna indican diferencias estadísticas según prueba de Duncan $(p<0,05)$. ND: No Detectada. $\pm S D$.

\begin{tabular}{|c|c|c|c|c|c|c|c|}
\hline Harina & $\begin{array}{c}\text { Treonina } \\
(\text { THR) }\end{array}$ & $\begin{array}{l}\text { Valina } \\
\text { (VAL) }\end{array}$ & $\begin{array}{l}\text { Isoleucina } \\
\text { (ILE) }\end{array}$ & $\begin{array}{l}\text { Leucina } \\
\text { (LEU) }\end{array}$ & $\begin{array}{c}\text { Fenilalanina } \\
+ \text { Tirosina } \\
\text { (PHE + TYR) }\end{array}$ & Lisina (LYS) & $\begin{array}{c}\text { Histidina } \\
\text { (HIS) }\end{array}$ \\
\hline Quinua & $1,65 \pm 0,02^{a}$ & $0,57 \pm 0,01^{a}$ & $0,64 \pm 0,01^{a}$ & $1,06 \pm 0,01^{a}$ & $0,52 \pm 0,01^{a}$ & $0,86 \pm 0,01^{a}$ & $0,49 \pm 0,02$ \\
\hline Chontaduro & $0,47 \pm 0,01^{b}$ & $0,21 \pm 0,01^{b}$ & $0,23 \pm 0,02^{b}$ & $0,41 \pm 0,01^{b}$ & $0,20 \pm 0,02^{b}$ & $0,32 \pm 0,02^{b}$ & ND \\
\hline
\end{tabular}

Las dos pastas STHQ y STHQHCh tienen en común la presencia de harina de quinua, cereal catalogado como fuente importante de proteínas debido a su digestibilidad y su composición equilibrada en aminoácidos esenciales (Danelli et al., 2012). La quinua presenta un contenido proteico igual o superior a otros cereales comunes como la propia sémola de trigo, las proteínas de la quinua radican principalmente en la albúmina y la globulina ricas en lisina (Mao et al., 2014).

Los altos porcentajes de proteínas que pueden tener los cereales de uso común para la elaboración de pastas alimenticias, no significa que algunos aminoácidos esenciales, como la lisina, metionina y valina estén presentes, normalmente este tipo de aminoácidos en los cereales comunes están en cantidades limitadas (Gil-Hernández, 2010; Gebremariam et al., 2014). El contenido de valina (VAL) fue diferente en las cuatro pastas secas evaluadas $(p<0,05)$, siendo la pasta STHQ la que exhibió el mayor valor con $1,55 \pm 0,01 \mathrm{~g} / 100 \mathrm{~g}$ de muestra. La lisina (LYS) es el aminoácido de mayor estudio e importancia en la composición nutricional de harinas y pastas alimenticias (Gil-Hernández, 2010; Mao et al., 2014), normalmente en los cereales comunes esta en cantidades inferiores a las requeridas para la nutrición humana (Mao et al., 2014). Las pastas secas STHQHCh y STHQ exhibieron los mayores contenidos de lisina, valores iguales a $\mathrm{p}<0,05$, de $0,45 \pm 0,02 \mathrm{~g} / 100 \mathrm{~g}$ de muestra y $0,44 \pm 0,02 \mathrm{~g} / 100 \mathrm{~g}$ de muestra respectivamente. La pasta seca ST denominada control o pasta comercial presentó el contenido de lisina más bajo, con un valor de 0,38 $\pm 0,01 \mathrm{~g} / 100 \mathrm{~g}$ de muestra, valor que relacionado con la pasta seca STHQHCh se observa que esta última presenta $15,6 \%$ más de contenido de lisina, consideración importante que sugierela importancia de la substitución de la harina de sémola de trigo por materias primas como la quinua y el chontaduro en las pastas alimenticias secas permitiendo aportar mayor calidad nutricional y en particular del aminoácido esencial lisina.

Tabla 5. Contenido de aminoácidos esenciales en las pastas secas extruidas ( $\mathrm{g} / 100 \mathrm{~g}$ de muestra). ST: sémola de trigo (control); STHQ: sémola de trigo y harina de quinua; STHCh: sémola de trigo y harina de chontaduro; STHQHCh: sémola de trigo, harina de quinua y harina de chontaduro. Diferentes letras minúsculas en la misma columna indican diferencias estadísticas según prueba de Duncan $(p<0,05)$. ND: No Detectada. $\pm S D$.

\begin{tabular}{|c|c|c|c|c|c|c|c|}
\hline Pasta & $\begin{array}{c}\text { Treonina } \\
(\mathrm{THR})\end{array}$ & $\begin{array}{l}\text { Valina } \\
\text { (VAL) }\end{array}$ & $\begin{array}{l}\text { Isoleucina } \\
\text { (ILE) }\end{array}$ & $\begin{array}{l}\text { Leucina } \\
\text { (LEU) }\end{array}$ & $\begin{array}{c}\text { Fenilalanina + } \\
\text { Tirosina (PHE } \\
+ \text { TYR) }\end{array}$ & Lisina (LYS) & $\begin{array}{c}\text { Histidina } \\
\text { (HIS) }\end{array}$ \\
\hline ST & $0,88 \pm 0,01^{a}$ & $1,53 \pm 0,01^{a}$ & $0,59 \pm 0,01^{a}$ & $1,12 \pm$ & $1,57 \pm 0,01^{a}$ & $0,38 \pm 0,01^{a}$ & ND \\
\hline STHQ & $0,94 \pm 0,01^{\mathrm{b}}$ & $1,55 \pm 0,01^{b}$ & $0,69 \pm 0,02^{b}$ & $1,18 \pm 0,01^{\mathrm{a}}$ & $1,83 \pm 0,02^{b}$ & $0,44 \pm 0,02^{\mathrm{bc}}$ & ND \\
\hline STHCh & $0.88 \pm 0,02^{a}$ & $1,43 \pm 0,01^{c}$ & $0,56 \pm 0,01^{c}$ & $1,03 \pm 0,01^{b}$ & $1,52 \pm 0,01^{c}$ & $0,43 \pm 0,01^{\mathrm{b}}$ & ND \\
\hline STHQHCh & $0.92 \pm 0,01^{\mathrm{c}}$ & $1,46 \pm 0,02^{d}$ & $0,58 \pm 0,01^{a}$ & $1,04 \pm 0,02^{b}$ & $1,39 \pm 0,02^{d}$ & $0,45 \pm 0,02^{\mathrm{c}}$ & ND \\
\hline
\end{tabular}

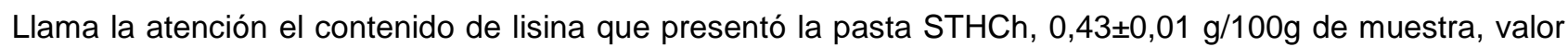
muy cercano al obtenido en las pastas con quinua, además la pasta STHCh había exhibido el menor contenido de proteína $(12,19 \pm 0,05 \mathrm{~g} / 100 \mathrm{~g}$ de muestra) (Tabla 3$)$ y el contenido de lisina muy superior de $0,86 \mathrm{~g} / 100 \mathrm{~g}$ de muestra en la harina de quinua en comparación con el contenido de lisina de $0,32 \mathrm{~g} / 100 \mathrm{~g}$ de muestra en la harina de chontaduro. Con estos datos, se esperaba que las pastas con contenido de harina de chontaduro resultaran con valores considerablemente menores de lisina que las pastas con quinua,pero no fue así. Seguramente en las pastas procesadas con harina de quinua se presentó una pérdida de lisina ocurrida por la reacción de Maillard y por el propio proceso de extrusión y secado. Cerón-Fernández et al. (2016) encontraron que el proceso de extrusión afecta el valor nutricional de las harinas de quinua de la variedad Blanca Jericó, la misma variedad aquí estudiada, encontrándose una disminución en los contenidos de proteína, fibra y grasa afectando las propiedades nutricionales de las pastas secas.

Camire (2002), explica que la Lisina es un aminoácido muy susceptible a las reacciones químicas por causa de las posiciones de los grupos amino, tendiendo a interactuar con los azúcares reductores presentes, favorecido igualmente por la acción mecánica. En la revisión de literatura, existe una cantidad limitada de 
información acerca de la perdida de nutrientes (Repo-Carrasco-Valencia et al., 2009),y la reacción de Maillard durante el proceso de extrusión de cereales (Masatcioglu et al., 2014).

Según Chao-Zhi et al. (2018) la histidina es un aminoácido esencial que participa en la reacción de Maillard en los alimentos. En este estudio solamente la harina de quinua presentó un contenido inicial de histidina con un valor de 0,49 $\pm 0,02 \mathrm{~g} / 100 \mathrm{~g}$ de muestra (Tabla 4), pero en el análisis de las pastas secas, este aminoácido no fue detectado, clara evidencia de la pérdida de este componente nutricional debido a su participación en la reacción de oscurecimiento no enzimático o Maillard y seguramente al proceso de extrusión.

\section{Análisis microbiológico}

El análisis microbiológico de las pastas extruidas secas se realizó para conocer el estado de inocuidad de los productos. El laboratorio externo que realizó los análisis utilizó la normatividad colombiana que rige en la actualidad y los microrganismos considerados fueron: recuento de mohos, recuento de levaduras, recuento de estafilococo, recuento de escherichia coli, recuento de bacillus cereus, detección de salmonella y recuento de esporas clostridium. Todos los resultados de recuentos y detección estuvieron por debajo de los valores máximos permitidos en la normatividad, es decir, todas las pastas secas eraninocuas.De acuerdo con Singh (2007) una de las ventajas que tienen las pastas alimenticias secas, es que debido al proceso de extrusión, el calor y las presiones actúan como factores de esterilización, eliminando o inactivación los microorganismos presentes. En caso que las pastas posean agentes patógenos, la cocción, o lo que es lo mismo, el tratamiento térmico de la pasta, elimina una parte muy significativa de los microorganismos que pudiera contener, como consecuencia, raramente estará implicada en procesos patológicos de origen microbiano.

\section{CONCLUSIONES}

De los resultados obtenidos del análisis proximal y del perfil de aminoácidos para la elaboración de pastas secas extruidas, se concluyó: 1. La sustitución de la harina de sémola de trigo por harinas de quinua y harina de chontaduro, son una alternativa de alimento con calidad proteica que cumple con porcentajes de proteína superiores al porcentaje mínimo establecido por la norma NTC 1055; 2. Las diferentes mezclas de harinas tuvieron un buen comportamiento en el extrusor, aunque se observó que el proceso termomecanico de extrusión incidió en las propiedades nutricionales finales de las pastas secas, siendo notoria la pérdida de aminoácidos y en particular de lisina.

\section{AGRADECIMIENTOS}

Los autores, agradecen a la Universidad Nacional de Colombia, Sede Palmira y a la Universidad del Cauca por los espacios cedidos para el desarrollo de la experimentación.

\section{REFERENCIAS}

AOAC: Association of Official Agricultural Chemists, AOAC 923.03, Amino Acids in Feeds, AOAC International Publisher, Gaithersburg, USA (1997).

AOAC: Association of Official Agricultural Chemists, AOAC 923.03, Ash of flour. 17 $7^{\text {th }}$ AOAC International Publisher,Gaithersburg, USA(2005).

AOAC: Association of Official Agricultural Chemists, AOAC 922.06, Fat in fl our, Acid hydrolysis method,19th Ed., AOACInternational, Gaithersburg, MD, USA(2012).

Astaiza, M., L. Ruiz y A. Elizalde,Elaboración de Pastas Alimenticias Enriquecidas a partir de Harina de Quinua (Chenopodium quinoa) y Zanahoria (Daucus Carota).ISSN 1692-3561, Biotecnología en el Sector Agropecuario y Agroindustrial, 8(1), 43-53 (2010).

Camire, M. E., Chemical and Nutritional Changes in Food During Extrusion. In: M. N. Riaz, Extruders in food applications, Boca Raton, FL: CRC Press, 127-148 (2002).

Cerón-Fernandez, C. L., L. V. Guerra-Morcilloy otros tres autores, Efecto de la Extrusión sobre las Características Físico-químicas de Harina de Quinua (Chenopodium quinoa Willd),doi: 10.18684/BSAA(14)92-99, Biotecnología en el Sector Agropecuario y Agroindustrial, 14 (2), 92-99 (2016).

Chao-Zhi, Z., Z. Jing-Li y otros cuatro autores, Contribution of Histidine and Lysine to the Generation of Volatile Compounds in Jinhua Ham Exposed to Ripening Conditions Via Maillard Reaction, doi: 10.1111/1750-3841.13996, Journal of Food Science, 83 (1), 46-52 (2018).

Chito-Trujillo, D. M., R. A. Ortega-Bonilla, A. F. Ahumada-Mamián y B. Rosero-López, Quinoa (Chenopodium quinoa Willd.) versus Soja (Glycine max [L.] Merr.) en la Nutrición Humana: Revisión sobre las Características Agroecológicas, de Composición y Tecnológicas, doi: 10.14306/renhyd.21.2.256, Spanish Journal of Human Nutrition and Dietetics, 21 (2), 184-198 (2017). 
Danelli, D., L. M. De Melo, S. H. Flôres y E. V. Jong, Chemical Analysis of Quinoa Flakes: Characterization for Use in Food Products, doi: http://dx.doi.org/10.1590/S1981-67232012005000023, Braz. J. Food Technol, 15 (4), $280-287$ (2012).

De Oliveira, M. K. S., H. E. Martinez-Flores, J. S. De Andrade, M. G. Garnica-Romo y, Y. K. Chang, Use of Pejibaye Flour (Bactris gasipaes Kunth) in the Production of Food Pastas, doi:10.1111/j.1365-2621.2005.01145.x, International Journal of Food Science and Technology, 41(8), 933-937(2006).

Departamento Nacional de Planeación,Bases del Plan Nacional de Desarrollo 2018-2022- Pacto por Colombia, Pacto por la Equidad. Bogotá D.C., Colombia: DNP (2018).

El-Bialee, N., A. M. Saad, M. I. El-Didamony y G. Zahran,Influence of Extrusion-Cooking Conditions on Corn Pasta Qualit, doi: 10.24018/ejers.2017.2.3.301, European Journal of Engineering Research and Science, 2(3), 24-29 (2017).

Firibu K., R. D. Saalia y S. Phillip,Degradation of Aflatoxins by Extrusion Cooking: Effects on Nutritional Quality of Extrudates, doi: 10.1016/j.Iwt.2011.01.021, LWT - FoodSci and Technol, 44(6), 1496-1501 (2011).

Gebremariam, M. M., M. Zarnkow y T. Becker, Teff (Eragrostis tef) as a Raw Material for Malting, Brewing and Manufacturing of Gluten-Free Foods and Beverages: A review, doi: 10.1007/s13197-012-0745-5, Journal of Food Science and Technology, 51(11), 2881-2895 (2014).

Gil-Hernández, A., Tratado de Nutrición:Composición y Calidad Nutritiva de los Alimentos,2a Ed., 812 pp, Editorial Médica Panamericana, Madrid(2010).

INCONTEC: Instituto Colombiano de Normas Técnicas y Certificación, NTC 668: Alimentos y materias primas. Determinación de los contenidos de grasa y fibra cruda, 5p, Bogotá, Colombia (1973).

INCONTEC: Instituto Colombiano de Normas Técnicas y Certificación, NTC 1055: Requisitos alimenticios de una pasta, 10p, Bogotá, Colombia (2007).

ICONTEC: Instituto Colombiano de Normas Técnicas y Certificación, NTC 529: Cereales y productos de cereales. Determinación del contenido de humedad, 18p, Bogotá, Colombia (2009).

INCONTEC: Instituto Colombiano de Normas Técnicas y Certificación, NTC 420: Productos de molinería. Sémola y semolato de trigo, 10p, Bogotá, Colombia (2015a).

INCONTEC: Instituto Colombiano de Normas Técnicas y Certificación. NTC 6116: Industrias alimentarias. Ovoproductos, 16p, Bogotá, Colombia (2015b).

ISO: International Organization for Standardization, ISO 1871: Food and Feed Products - General Guidelines for the Determination of Nitrogen by the Kjeldahl Method. Second edition, 7p, Suiza (2009).

Liting W., L. Lin y otros cuatro autores, Effect of Barrel Temperature and Moisture Content on the Composition and Oxidative Stability of Extruded Palm Oil in an Oil-Starch Model System,doi: https://doi.org/10.1016/j.Iwt.2018.08.019, LWT - Food Science and Technology, 98(1), 398-495 (2018).

Maldonado, P.,Embutidos Fortificados con Proteína Vegetal a Base de Quinua (Chenopodium quinoa Wild.), doi: https://doi.org/10.29019/enfoqueute, Enfoque UTE, 1(1), 36 - 45 (2010).

Manthey, F. A., S. R. Yalla, T. J. Dick y M. Badaruddin, Extrusion Properties and Cooking Quality of Spaghetti Containing Buckwheat Bran Flour, doi:10.1094/cchem.2004.81.2.232, Cereal Chemistry Journal, 81(2), 232-236 (2004).

Mao, X., Y. Hua y G. Chen, Amino Acid Composition, Molecular Weight Distribution and Gel Electrophoresis of Walnut (Juglans regia L.) Proteins and Protein Fractionations, doi: 10.3390/ijms15022003. International Journal of Molecular Sciences, 15, 2003-2014 (2014).

Masatcioglu, M. T., V. Gokmen, P. K. W. Ng y H. Koksel, Effects of Formulation, Extrusion Cooking Conditions, and $\mathrm{CO}_{2}$ Injection on the Formation of Acrylamide in Corn Extrudates, doi: https://doi.org/10.1002/jsfa.6598, J. Sci. Food Agric. 94, 2562-2568 (2014).

Murphy, K. y J. Matanguihan, Quinoa: Improvement and Sustainable Production, John Wiley \& Sons, Inc., doi: 10.1002/9781118628041, 235pp, New Jersey, Estados Unidos (2015).

Repo-Carrasco-Valencia, R., J. Pena, H. Kallio y S. Salminen, Dietary Fiber and Other Functional Components in two Varieties of Crude and Extruded Kiwicha (Amaranthus caudatus), doi: https://doi.org/10.1016/j.jcs.2008.10.003, J. Cereal Sci. 49, 219-224 (2009).

Restrepo, J. R., L. E. Vinasco y J. A. Estupiñán, Estudio Comparativo del Contenido de Ácidos Grasos en 4 Variedades de Chontaduro (Bactris gasipaes) de la Región del Pacífico Colombiano, ISSN 2248-4000, 16, 123-129 (2012).

Serpa-Guerra, A. M., L. M. Vélez Acosta y otros tres autores, Compuestos de hierro para la fortificación de alimentos: El desarrollo de una estrategia nutricional indispensable para países en vía de desarrollo, doi: https://dx.doi.org/10.15446/acag.v65n4.50327, Una revisión. Acta Agronómica, 65(4), 340-353 (2016).

Singh S., S. Gamlath y L. Wakeling, Nutritional Aspects of Food Extrusion: A Review, doi: https://doi.org/10.1111/j.13652621.2006.01309.x, Int J Food Sci and Technol., 42(8), 916-929 (2007).

Wójtowiez, A.,Influence of Process Conditions on Selected Texture Properties of Precooked Buckwheat Pasta, TEKA-commission of motorization and energetics in agriculture, ISSN: 1641-7739, 12(1), 315-322 (2012). 